\title{
Metanephric adenoma: association between the imaging features of contrast-enhanced ultrasound and clinicopathological characteristics
}

\author{
Dongmei Zhu ${ }^{1,2 \#}$, Lin $\mathrm{Zhu}^{2,3 \#}$, Ji Wu${ }^{4}$, Linghu $\mathrm{Wu}^{1}$, Jian $\mathrm{Li}^{1}$, Hao Luo ${ }^{2}$, Keen Yang ${ }^{1}$, Gang Liu ${ }^{2}$, \\ Xiaoguang Quo ${ }^{5}$, Fajin Dong ${ }^{1}$, Jinfeng Xu ${ }^{1}$
}

${ }^{1}$ Department of Ultrasound, The Second Clinical Medical College, Jinan University (Shenzhen People's Hospital), Shenzhen, China; ${ }^{2}$ Department of Ultrasound, The Affiliated Nanchong Central Hospital of North Sichuan Medical College, Nanchong, China; ${ }^{3}$ Department of Geriatric General Surgery, The First Affiliated Hospital of Kunming Medical University, Kunming, China; ${ }^{4}$ Department of Urology Surgery, The Affiliated Nanchong Central Hospital of North Sichuan Medical College, Nanchong, China; ${ }^{5}$ Department of Pathology, The Affiliated Nanchong Central Hospital of North Sichuan Medical College, Nanchong, China

Contributions: (I) Conception and design: D Zhu, L Zhu; (II) Administrative support: F Dong, J Xu; (III) Provision of study materials or patients: J Wu, L Wu, H Luo, K Yang; (IV) Collection and assembly of data: J Li, G Liu, X Quo; (V) Data analysis and interpretation: D Zhu, L Zhu; (VI) Manuscript writing: All authors; (VII) Final approval of manuscript: All authors.

\#These authors contributed equally to this work.

Correspondence to: Jinfeng Xu; Fajin Dong. Department of Ultrasound, The Second Clinical Medical College, Jinan University (Shenzhen People's Hospital), 1017 Dongmen North Road, Luohu District, Shenzhen 518020, China. Email: xujinfeng@yahoo.com; dongfajin@szhospital.com.

Background: This study aimed to improve the understanding of metanephric adenoma (MA) by retrospective analysis of contrast-enhanced ultrasound (CEUS) findings and clinicopathological characteristics of MAs.

Methods: Gray-scale ultrasound (US) and CEUS findings of 7 adult MA patients, confirmed by postoperative pathology, were summarized via collection of clinicopathological and ultrasonographic imaging data, including tumor location, size, echo intensity, color flow, presence or absence of calcification, and liquefactive necrosis, contrast-enhanced pattern, enhancement characteristics, and contrast wash-out compared with adjacent parenchyma, and the presence or absence of a pseudocapsule. Histopathological analyses, including hematoxylin and eosin (HE) and immunohistochemical (IHC) staining, were conducted with the EnVision method.

Results: All 7 participants were female, aged 29-73 years (mean age, 54 years), with flank pain (3/7). All tumors were solid (7/7) with sizes of 2.0-5.0 cm (mean diameter, $3.07 \mathrm{~cm}$ ), including 4 in the left kidney, 3 in the right kidney, 2 in the renal pelvis, and 5 in the renal parenchyma. On the gray-scale US, MA was shown as hypoechoic (4/7), slightly hyperechoic (2/7), isoechoic (1/7), and with a defined border. The morphology was regular and rounded (7/7), internal echogenicity was homogeneous (5/7), and no calcification was seen (7/7). The CEUS showed clear boundaries (7/7), homogeneous isodensity (5/7), with calcification $(0 / 7)$, necrosis (2/11), heterogeneous hyperattenuation $(2 / 7)$, pseudocapsule $(2 / 7)$, and medullary phase fast washout (7/7). The surgical methods were radical nephrectomy (4/7) and partial nephrectomy (3/7). The duration of follow-up period for all participants was 3-74 months, and no local or distant recurrences were found. The IHC staining showed that most tumor cells were positive for WT1, cytokeratins AE1/AE3, vimentin, and CD57, and exhibited focal positivity for CK7, while negative for CD10, AMACR, and CK720. The proliferative index (Ki-67) was 2-3\%.

Conclusions: On gray-scale US, MA appears as a solid nodule with a well-defined boundary, regular morphology, and homogeneous echogenicity; CEUS shows slow progression and slightly lower homogeneous enhancement and fast wash-out in the medullary phase. These findings may provide insight into the progression of MA and aid in the development of diagnostic and therapeutic strategies. 
Keywords: Kidney; metanephric adenoma (MA); contrast-enhanced ultrasound (CEUS); hypovascular kidney neoplasm

Submitted Jun 17, 2021. Accepted for publication Aug 12, 2021.

doi: $10.21037 /$ gs-21-437

View this article at: https://dx.doi.org/10.21037/gs-21-437

\section{Introduction}

Metanephric adenoma (MA) is a rare benign tumor that was classified in 2016 by the World Health Organization (WHO) as a renal tumor (1). The histological origin of MA is still under debate, but it is believed to originate from a metanephric blastoma, which is characterized by an admixture of tiny, elongated, tightly packed tubules and spindle cells present in a mucinous background. Most MAs are benign tumors, which are frequently misdiagnosed as malignant, resulting in overtreatment or unnecessary radical nephrectomy surgery. Therefore, a precise pathological diagnosis before surgery is essential. Approximately 100 MA cases have been reported by computed tomography (CT) and magnetic resonance imaging (MRI) so far $(2,3)$. However, little research on contrast-enhanced ultrasound (CEUS) imaging features of MA has been reported to date. We retrospectively analyzed the imaging features of CEUS of MA based on 7 cases of MA and performed a literature review to increase understanding of this disease.

We present the following article in accordance with the STROBE reporting checklist (available at https://dx.doi. org/10.21037/gs-21-437).

\section{Methods}

\section{Patients}

We obtained the information for 7 cases diagnosed as MA from 2014 to 2020 from the surgical pathology database of the Pathology Department of The Affiliated Nanchong Central Hospital of North Sichuan Medical College and The Second Clinical Medical College of Jinan University. Patients' data were collected retrospectively, including age, gender, clinical manifestations, preoperative tumor characteristics (size, location), and final pathological findings (Table 1).

The study was approved by the Ethics Committee of The Second Clinical Medical College, Jinan University (Shenzhen People's Hospital) (18PJ149). All procedures were conducted in accordance with the principles of the
Declaration of Helsinki (as revised in 2013). Individual patient information was protected and has not been shown. Individual consent for this retrospective analysis was waived.

\section{Pathologic examination}

All 7 participants underwent tumor resection. Cystic components and metastasis of tumors were assessed intraoperatively. Pathological diagnosis of MA was made according to the latest WHO criteria. Tumor samples were fixed with $10 \%$ formaldehyde, and routine paraffin embedding was performed. Tumor size was evaluated using caliper measurements. The shape, location, cystic components, and capsule formation of tumors was observed with the naked eye. Histopathological analyses, including hematoxylin and eosin (HE) and immunohistochemical (IHC) staining, were performed to assess tumor components, including the percentage of tubules, spindle cells, and myxoid stroma via the ELPS (Enhance Labeled Polymer System, Dako, Agilent Technologies, USA) method.

\section{Imaging technique and analysis}

All the CEUS examinations were conducted by the same doctor, who had 7 years' experience in abdominal ultrasound (US) and CEUS and was blinded to the diagnosis, with the LOGIC E9 system (GE Healthcare, Wauwatosa, WI, USA; C1-5, 1-5 MHz). An abdominal US was performed first. Doppler parameters were optimized to detect slow flow velocities with a pulse repetition frequency of $700 \mathrm{~Hz}$, medium wall filter, and low-velocity flow optimization. First, conventional US was conducted to localize the mass in cross-sections and longitudinal sections, including the normal renal cortex adjacent to the mass. Next, the single-focus was placed below the mass, while the depth, overall gain, time gain compensation, and compression were optimized. In all CEUS examinations, the contrast agent SonoVue ${ }^{\circledR}$ (Bracco, Milan, Italy) was used. In most 
Table 1 Clinical features of 7 MA patients

\begin{tabular}{|c|c|c|c|c|c|c|c|c|c|c|}
\hline Patient & $\begin{array}{l}\text { Age } \\
\text { (years) }\end{array}$ & Size $(\mathrm{cm})$ & Side & $\begin{array}{l}\text { Mass location } \\
\text { in kidney }\end{array}$ & $\begin{array}{c}\text { Surgery method } \\
\text { RN/PN }\end{array}$ & Metastasis & $\begin{array}{l}\text { Follow-up } \\
\text { (months) }\end{array}$ & \multicolumn{3}{|c|}{ Clinical symptoms } \\
\hline$\# 1$ & 45 & $2.0 \times 2.0$ & L & Renal edulla & $\mathrm{RN}$ & $\mathrm{N}^{*}$ & 74 & $\mathrm{Y}$ & $\mathrm{N}$ & $\mathrm{N}$ \\
\hline \#2 & 73 & $3.0 \times 2.0$ & $\mathrm{R}$ & Renal pelvis & $\mathrm{RN}$ & $\mathrm{N}^{*}$ & 60 & $\mathrm{Y}$ & $\mathrm{N}$ & $\mathrm{N}$ \\
\hline \#3 & 60 & $5.0 \times 4.0$ & $\mathrm{R}$ & Renal edulla & $\mathrm{RN}$ & $\mathrm{N}^{*}$ & 52 & $\mathrm{~N}$ & $\mathrm{~N}$ & $\mathrm{~N}$ \\
\hline \#5 & 29 & $3.0 \times 3.0$ & $\mathrm{R}$ & Renal pelvis & PN & $\mathrm{N}^{*}$ & 20 & $\mathrm{~N}$ & $\mathrm{~N}$ & $\mathrm{~N}$ \\
\hline \#6 & 66 & $2.5 \times 2.5$ & L & Renal edulla & RN & $\mathrm{N}^{*}$ & 19 & $\mathrm{~N}$ & $\mathrm{~N}$ & $\mathrm{~N}$ \\
\hline$\# 7$ & 52 & $5.0 \times 4.5$ & L & Renal edulla & PN & $\mathrm{N}^{*}$ & 3 & $\mathrm{~N}$ & $\mathrm{~N}$ & $\mathrm{~N}$ \\
\hline
\end{tabular}

$\mathrm{N}^{*}$ indicates no metastasis. MA, metanephric adenoma; R, right; L, left; RN, radical nephrectomy; PN, partial nephrectomy; $\mathrm{Y}$, present, $\mathrm{N}$, nothing.

cases, $1.0-1.2 \mathrm{~mL}$ (min., $1.0 \mathrm{~mL}$; max., $5.0 \mathrm{~mL}$ ) contrast agent was injected as a bolus injection by a peripheral 18-22 G needle followed by a flush of $5-10 \mathrm{~mL}$ of $0.9 \%$ sodium chloride solution. In most instances, a single dose of contrast agent met requirements for diagnosis; in individual cases, it was repeated up to 2 times. The CEUS was sustained for at least 2-3 min. In our research, we used the following phase terms: cortical phase, which began $10-15 \mathrm{~s}$ after injection until 30-45 s, and medullary phase, approximately $30-45 \mathrm{~s}$ after injection until the microbubble echoes disappeared entirely. The entire course of CEUS was captured and saved on a hard disk in Digital Imaging and Communication in Medicine (DICOM) format for documentation and subsequent qualitative analysis. The videos were retrospectively analyzed by two doctors (with 6 and 8 years of experience in CEUS imaging), independently blinded to clinical and pathologic information. Location, size, echogenicity, border, shape, and vascularization of the lesions were appraised via conventional US. The definitions of enhancement patterns and characteristics of CEUS were as follows: (I) at peak enhancement of masses, depending on the degree of enhancement of the mass compared with that of the renal cortex, hyper, iso-, and hypo-enhancement were defined; (II) homogenous enhancement was defined as a mass occupied by complete enhancement without any defects. Heterogeneous enhancement was defined as a mass with unenhanced areas, regardless of diverse degrees of enhancement; (III) in terms of washout patterns, "fastwashout", "synchronous-out", and "slow-out" indicated that the outflow of the contrast agent from the mass was faster than, simultaneous with, and slower than that from the adjacent cortex, respectively; (IV) a rim of perilesional enhancement after enhancement agent injection was considered a pseudocapsule.

\section{Statistical analysis}

All data were analyzed using SPSS ${ }^{\circledR}$ v.17 (SPSS Inc., Chicago, IL, USA). A P value $<0.05$ was considered to be statistically significant. The observed results consistency between the two doctors was tested by calculating the interclass correlation coefficient (ICC).

\section{Results}

\section{Clinicopathological characteristics}

All 7 participants were female, aged 29-73 years (mean age, 54 years), with flank pain (3/7) and lesions (4/7) identified during normal physical examination. Tumor sizes were $2.0-5.0 \mathrm{~cm}$ (mean diameter, $3.07 \mathrm{~cm}$ ), including 4 in the left kidney, 3 in the right kidney, 2 in the renal pelvis, and 5 in the renal parenchyma; all tumors were solid (7/7). Details are displayed in Table 1.

The gross visual examinations found that tumors were gray-yellow or gray-white. Light microscopy showed that tumor cells were abundant, arranged in dense, indistinct stroma, with small and uniform cells arranged in an acinar or papillary pattern. The tumor cells were monomorphic with small and round nuclei, inconspicuous nucleoli, and delicate chromatin. There was scanty cytoplasm and pale pink staining; karyorrhexis was rare and cellular atypia was not evident (Figure 1A,1B). Immunostaining revealed that 7 

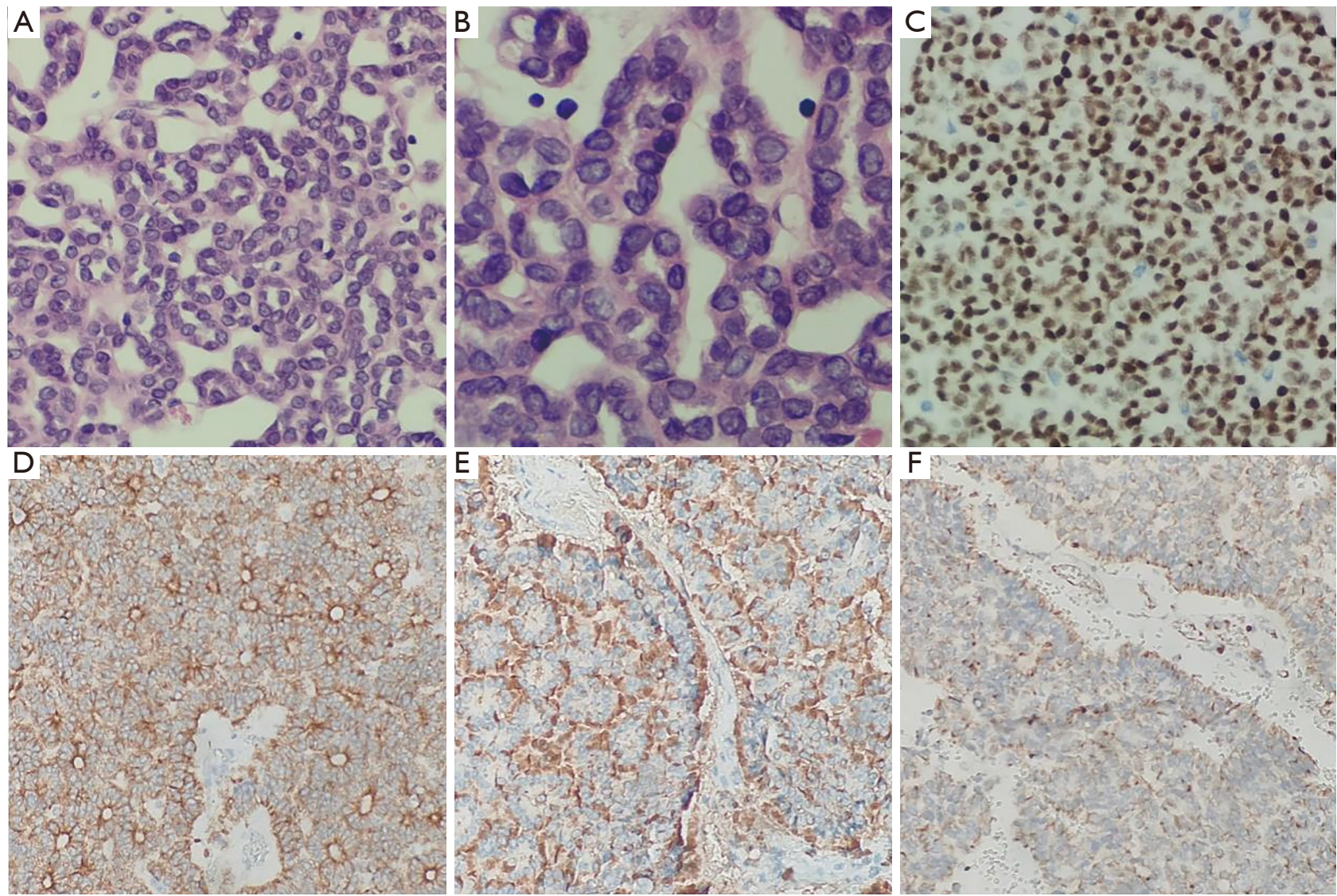

Figure 1 Pathological characteristics of MA. (A) HE staining. Magnification, $\times 200$. Tumor cells are epithelioid-like and arranged in cords. (B) HE staining. Magnification, $\times 400$. Tumor cells are round or oval. (C-F) IHC staining. Magnification, $\times 200$. Tumor cells are positive for WT1, PCK, CD57, and vimentin, respectively. MA, metanephric adenoma; HE, hematoxylin and eosin; IHC, immunohistochemical.

samples were positive for WT1, PCK, vimentin, and CD57 (Figure $1 C-1 F$ ), negative for EMA and CK7, and exhibited low expression of $\mathrm{Ki}-67(<10 \%)$. However, none of the 7 cases were tested for BRAF V600E mutation, and it is unknown whether there was a genetic mutation.

\section{Gray-scale US and CEUS findings}

The conventional US showed that all 7 lesions were predominantly hypoechoic. Tumors were solid (7/7), with a clear boundary (7/7); hypoechoic (4/7), isoechoic (1/7), or slightly hyperechoic (2/7) (Figure 2A-2C), with homogeneous internal echogenicity (5/7), and no calcification was seen (7/7). Color Doppler flow imaging (CDFI) manifested no obvious blood flow into the 7 lesions, but some blood flowed around them (Figure 2D). As the kidney is a richly perfused organ, tumor blood flow equal to or greater than cortical blood flow was defined as hypervascular, tumor blood flow less than cortical blood flow as hypovascular, and the absence of tumor blood flow as avascular.

\section{CEUS findings}

There was good agreement between the two doctors regarding enhancement characteristics, as evidenced by $\kappa$ statistics that ranged from 0.81 to 0.92 (Table 2). After the use of contrast medium, we found less enhancement of the 7 masses compared with the adjacent normal renal cortex $(7 / 7)$ than that of the renal, rapid clearance of the medullary phase (7/7), and enhanced homogeneous (5/7), pseudocapsule (2/7), and some liquefactive necroses were seen within masses (2/7). The CEUS manifested less blood flow in all the MA lesions than renal parenchyma 

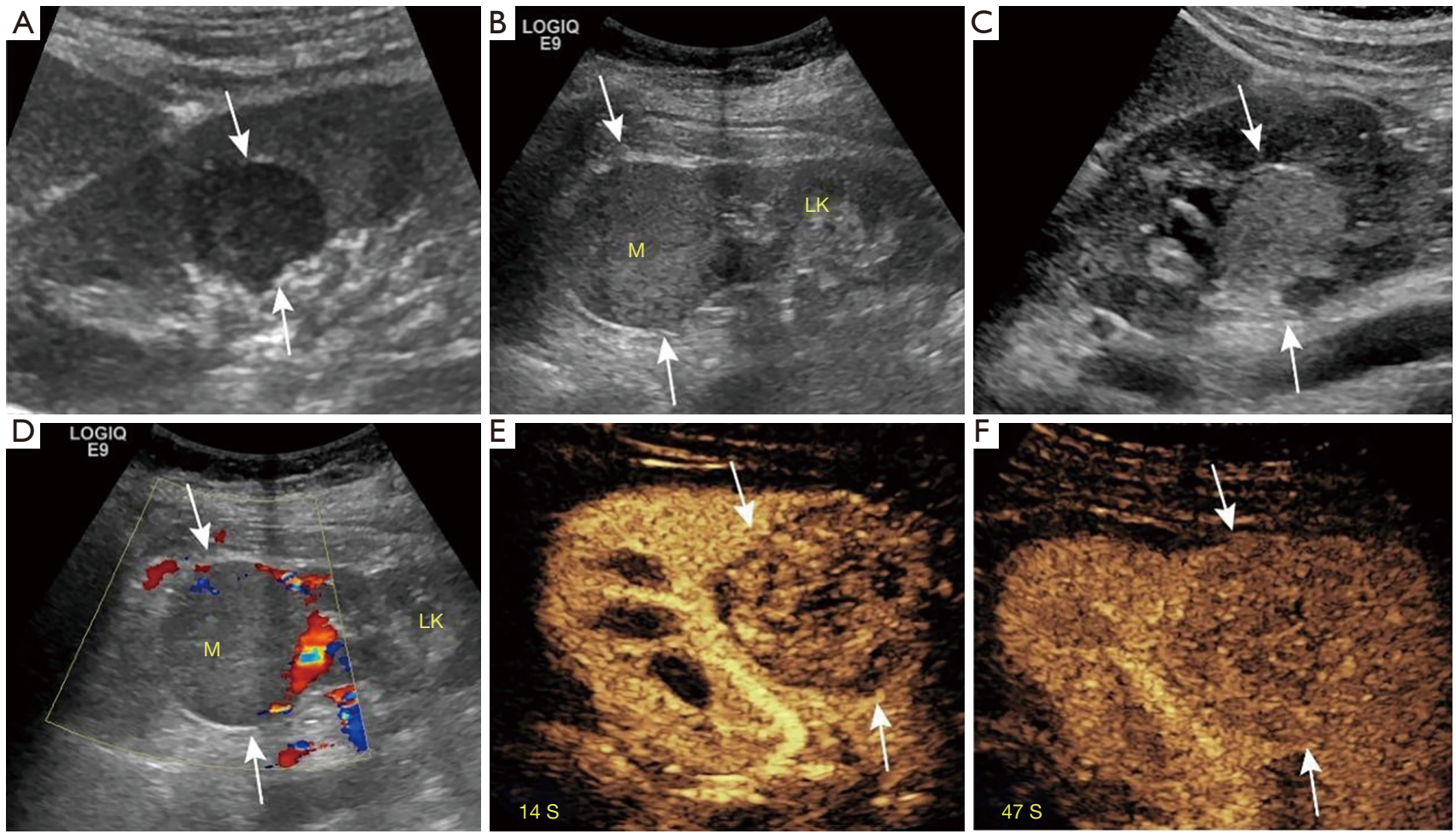

Figure 2 Conventional US and CEUS features of MA. The white arrows indicate the mass. (A-C) Conventional US showed well-defined hypoechoic, isoechoic, and slightly hyperechoic masses, respectively. (D) CDFI indicated some blood flowed around the mass. (E,F) CEUS showed that the enhancement of MA was lower than that of the adjacent normal renal cortex and medulla during all enhanced phases. US, ultrasound; CEUS, contrast-enhanced ultrasound; MA, metanephric adenoma; CDFI, color Doppler flow imaging; M, mass; LK, left kidney.

(Figure 2E,2F), indicating hypovascular renal tumors. The enhancement was lower in the MA than in the renal cortex and medulla during all enhanced phases. We defined these tumors as hypovascular renal masses via CEUS. In all masses, the enhancement pattern manifested as "lightly slow wash-in" and "fast wash-out". Gray-scale US and CEUS findings are shown in Tables 2,3.

\section{Preoperative diagnosis}

Of the 7 cases, 6 were misdiagnosed preoperatively as renal cell carcinoma (RCC), while 1 was diagnosed as an emerging renal tumor under CEUS by reviewers.

\section{Therapy and follow-up}

The surgical methods used were radical nephrectomy (4/7) and partial nephrectomy (3/7), and the follow-up period ranged from 3 to 74 months (mean, 32.7 months). All participants were alive and well during the followup period. All 7 participants showed no relapses via abdominal CEUS and/or contrast-enhanced computed tomography (CECT) examination at 3, 6, and 12 months, and then annually after surgery.

\section{Discussion}

A rare benign renal tumor with specific histopathological features, MA has an incidence of $0.2 \%$ among renal epithelial tumors. Metanephric adenofibroma, and metanephric stromal tumor were uniformly called MA by the WHO in 2016, which had rarely reported regional lymph node and distant metastasis and death (4). Due to low incidence, there have been few reports of MA diagnosis and differential diagnosis with conventional US and CEUS. 


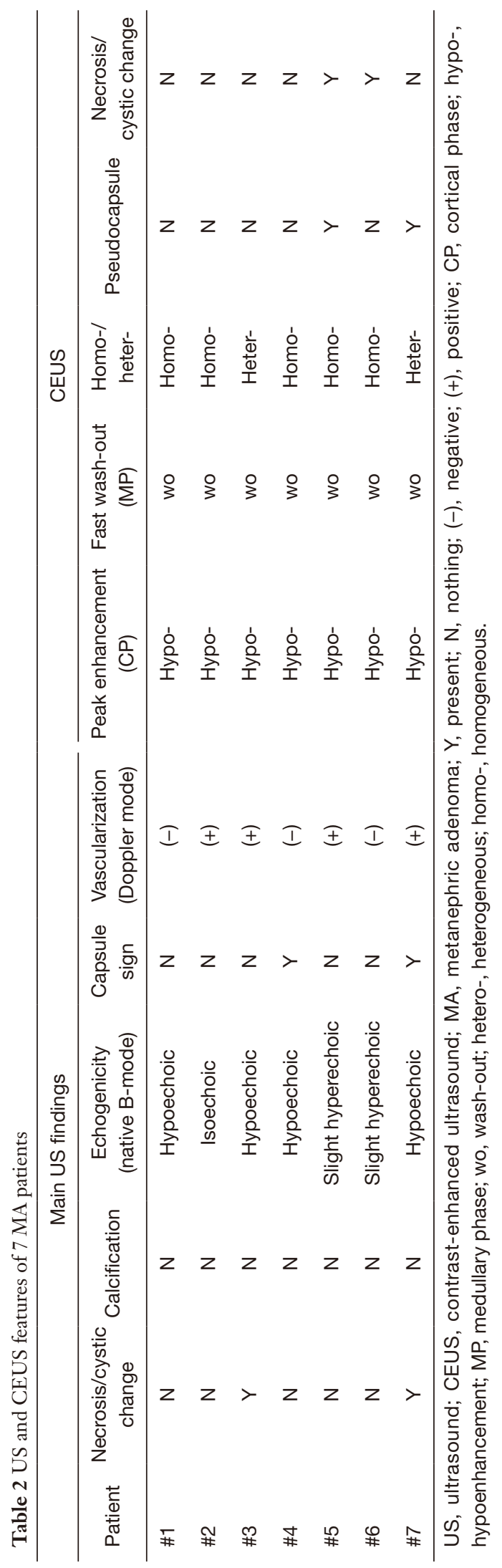

Table 3 The ICC between two readers and 95\% CI of CEUS features

\begin{tabular}{lll}
\hline Features & ICC & $95 \% \mathrm{Cl}$ \\
\hline Necrosis & 0.83 & 0.76 to 0.87 \\
Peak enhancement & 0.89 & 0.81 to 0.94 \\
Wash-out & 0.81 & 0.70 to 0.88 \\
Homogeneity & 0.92 & 0.84 to 0.96 \\
Pseudocapsule & 0.87 & 0.79 to 0.92
\end{tabular}

ICC, interclass correlation coefficient; Cl, confidence interval; CEUS, contrast-enhanced ultrasound.

\section{Clinicopathological characteristics}

A wide range of patients are affected by MA, and the majority are female (male to female ratio of 1:2), with occasional occurrence in children (5). Patients with MA usually present asymptomatically and MA is detected by US, but it may present with flank pain, mass, and hematuria. In this work, 4 cases exhibited flank pain without hematuria and polycythemia. A previous case report of MA in a child described growth from 0.7 to $3.0 \mathrm{~cm}$ in 5 years. Davis et al. found that $50 \%$ of cases were incidental findings and most were reported without clinical symptoms (3). The participants in this group were all female and only 3 patients presented with slight lumbar discomfort, no hematuria, and erythrocytosis, which may have been associated with small tumor size or too few samples.

As little is known about its specific pathogenesis, some academics uphold that MA originates from the residual tissue of renal cortical tubules during embryonic development of the kidney. Histologically, the typical characteristics of MA cover an interconnecting tubular, spindled architecture within the myxoid stroma and lowgrade nuclei, areas of necrosis and hemorrhagic, solid tubular growth, and foam cell deposits. It has been shown that MA exhibits variable immunoreactivity for keratins, including the cytokeratins AE1/AE3, CD57, vimentin, S-100, epithelial membrane antigen, and WT1 (6). In spite of some variability, MA is often focally positive for cytokeratin (7). There have been few cytogenetic and molecular genetic studies conducted on MA. The vast majority of reports have involved single cases. From a genetic aspect, $\mathrm{MA}$ is a distinct and independent entity, in spite of morphologic and immunophenotypic similarities to papillary renal cell carcinoma (pRCC), renal adenoma, 
and Wilms tumor $(7,8)$. Although difficult to distinguish from other tumors, including Wilms tumor and pRCC, which show similar cytologic features, gathering clinical information, IHC, and cytogenetic researches is worthwhile.

\section{Imaging with US and CEUS}

Conventional US characteristics of MA have been reported in a few cases as isoechoic, hypoechoic, or slightly hyperechoic with homogeneous internal echogenicity (9), and color Doppler has generally shown no blood flow signal inside the tumor or a few blood flow signals in the periphery. In our cases, 4 lesions were hypoechoic, 1 was isoechoic, 2 were slightly hyperechoic, and the internal echogenicity was homogeneous; a little hemorrhagic necrotic change was seen in 2 tumors, both of which were larger than $5 \mathrm{~cm}$ in diameter. Bastide et al. reported MA as well-circumscribed, usually hyperechoic solid masses on US (10). Fielding et al. implied that the hyperechogenicity might be due to psammomatous calcifications and interfaces caused by numerous tubules (11). Some liquefactive necrosis is thought to occur when tumors are larger (12). In our study, the overall enhancement pattern revealed by CEUS was slightly later than that in the renal cortex and the peak enhancement was lower than that in the surrounding normal renal cortex; 5 lesions showed homogeneous enhancement, 2 larger lesions showed less heterogeneous enhancement, and tumors in the medullary phase showed rapid wash-out. A pseudocapsule around the tumor was detected in 2 lesions, with 1 located in the renal pelvis and the other located in the renal medulla. These enhancement characteristics might be related to their histological structure composition. As histopathology indicates that tumor cells have tubular, glandular, and papillary patterns, stroma, and sparse vascular numbers, it determined the characteristics of tumor vessels. Papillary hyperplasia might show marked enhancement when it is more usual in MA and more capillaries are present. Unlike previous reports, no calcifications were found in and around the 7 lesions. This pattern is different from tumors with high attenuation solid masses, covering clear cell renal cell carcinomas (ccRCC), papillary carcinomas, angiomyolipomas with minimal fat, and renal oncocytoma $(13,14)$.

\section{Diagnosis and differential diagnosis of $M A$ with imaging}

Due to its safety, real-time approach, simplicity, patient tolerance, lack of radiation, and increased sensitivity compared to CT, CEUS has been widely used in depicting microvessels in tumors, perfect presentation of blood distribution and flow, and prompt potential diagnostic value (15). It is essential that ccRCC be considered in the differential diagnosis of MA at first; ccRCC is the most prevalent type among malignant tumors of the kidney (90\% of all renal tumors). Due to the rich blood supply of ccRCC, rapid heterogeneous hyperenhancement in the typical CEUS pattern and a pseudocapsule can be seen in the periphery. Sonographic contrast-specific imaging is visualized with a tumoral pseudocapsule, which is a characteristic performance. This finding could be useful in sonographic diagnosis of RCC (16).

However, MA is a tumor with poor blood supply, and the internal enhancement pattern and enhancement characteristics are significantly different; thus, differential diagnosis is easier between the 2 diseases. The point of difficulty lies in the differentiation between hypovascular renal tumors, including pRCC, chromophobe renal cell carcinoma (cRCC), and poor fat renal angiomyolipoma, as well as Wilms carcinoma. There have been few previous reports on CEUS of MA. In our work, CEUS findings for the 7 lesions were identical, and showed slow hyperenhancement and rapid medullary phase clearance. Due to the rarity of this condition and lack of awareness of its sonographic features, 6 cases in this group had been preoperatively diagnosed as malignant renal tumors, and 1 with a benign renal tumor. The rate of visible misdiagnosis was quite high. A paper by Xue $e t a l$. reported 200 cases of ccRCC. In small lesions with a diameter $\leq 3 \mathrm{~cm}$, most of the 3 subtypes of RCC showed homogeneous enhancement, and there was no difference among them (17). Most pRCC and chRCC manifested hypoenhancement, homogeneity, fast wash-out, and a pseudocapsule. The only difference between pRCC and chRCC was the wash-in pattern, with slow wash-in in pRCC and simultaneous wash-in in chRCC. Mueller-Peltzer et al. analyzed the varying enhancement patterns of pRCC and chRCC in 60 cases; $75 \%$ of pRCC cases manifested typical hypoenhancement and wash-out. However, $25 \%$ of pRCC cases showed an atypical enhancement pattern making it hard to distinguish from other kidney masses (18). Quantitative analysis of more cases with CEUS is required.

The imaging characteristics of adrenal myelolipoma (AML) and RCC have many overlaps. Lipid-poor AMLs can have isoechoic or mildly hyperechoic ultrasonographic findings. Moreover, RCC can be uniformly hyperechoic 
on US and resemble the appearance of AML. Kazmierski et al. reported that $32 \%$ of small renal tumors $(<3 \mathrm{~cm}$ in diameter) were sufficiently hyperechoic to resemble AML $(19,20)$. However, Xu et al. analyzed 84 RCCs by CEUS, and found that $88.1 \%$ of RCCs manifested hyperenhancement in the cortical phase (21). Therefore, there is a significant relationship between the size of the lesion and the enhancement characteristics. The smaller the lesion, the more difficult it is to diagnose. Wilms tumor is found predominantly in children aged 3-4 years; it is the most prevalent malignant tumor of the abdomen in children. The mass has different echogenicity, density, or intensity on US, indicating hemorrhage, mildness, necrosis, or calcification. We must always consider MA in the differential diagnosis of renal tumors, especially when the tumors appear as well-defined rounded masses.

With the availability of needle biopsy techniques, performing a pathological biopsy by preoperative needle aspiration for definitive diagnosis when MA is suspected based on clinical findings and imaging studies may increase the likelihood of renal sparing, with more significant benefit for patients whose tumors are highly considered for radical nephrectomy. The treatment of MA is still primarily surgery, with a surgical approach preferred to enucleation or partial nephrectomy, in addition to selection of the procedure according to tumor size, location, medical conditions, and experience and level of the operating surgeon. As MA is a benign tumor, the prognosis is better following complete resection of the tumor. No recurrence or metastasis of tumors occurred among the participants in this study, while there have been reports of individual MA patients developing pulmonary, bone, and lymph node metastases after surgery. Besides, MA manifested slight homogeneous enhancement on CEUS compared to normal renal parenchyma. Even slight blood flow on contrast studies of these masses could prompt doctors to question a hypovascular tumor, resulting in surgical resection.

\section{Limitations}

This was a retrospective study, which may have resulted in selective bias. Only 7 masses were enrolled in this study; thus, the sample of MAs was relatively small. Finally, this study did not use the quantitative evaluation analysis tool of CEUS; this novel method could not be recognized objectively. A larger sample size is needed for further investigation and validation of our findings.

\section{Conclusions}

The renal disease MA is a solid nodule with a clear boundary, regular morphology, and homogeneous echogenicity on gray-scale US. Use of CEUS showed slow progression and slightly lower homogeneous enhancement and fast wash-out in the medullary phase. These findings may provide insight into the progression of MA and aid in the development of diagnostic and therapeutic strategies for MA.

\section{Acknowledgments}

We would like to thank all of our colleagues from The Affiliated Nanchong Central Hospital of North Sichuan Medical College and our Dream Team of Ultrasound, The Second Clinical Medical College of Jinan University. We thank International Science Editing (http://www. internationalscienceediting.com) for editing this manuscript. Funding: The work described in this paper was supported by the Natural Science Foundation of Shenzhen (JCYJ20190806151807192) and the National Natural Science Foundation of China (81771841).

\section{Footnote}

Reporting Checklist: The authors have completed the STROBE reporting checklist. Available at https://dx.doi. org/10.21037/gs-21-437

Data Sharing Statement: Available at https://dx.doi. org/10.21037/gs-21-437

Conflicts of Interest: All authors have completed the ICMJE uniform disclosure form (available at https://dx.doi. org/10.21037/gs-21-437). All authors reported that this work was supported by the Natural Science Foundation of Shenzhen (No. JCYJ20190806151807192) and the National Natural Science Foundation of China (No. 81771841). The authors have no other conflicts of interest to declare.

Ethical Statement: The authors are accountable for all aspects of the work in ensuring that questions related to the accuracy or integrity of any part of the work are 
appropriately investigated and resolved. All procedures performed in this study involving human participants were in accordance with the Declaration of Helsinki (as revised in 2013). The study was approved by the Ethics Committee of The Second Clinical Medical College, Jinan University (Shenzhen People's Hospital) (18PJ149). Individual consent for this retrospective analysis was waived.

Open Access Statement: This is an Open Access article distributed in accordance with the Creative Commons Attribution-NonCommercial-NoDerivs 4.0 International License (CC BY-NC-ND 4.0), which permits the noncommercial replication and distribution of the article with the strict proviso that no changes or edits are made and the original work is properly cited (including links to both the formal publication through the relevant DOI and the license). See: https://creativecommons.org/licenses/by-ncnd $/ 4.0 \%$.

\section{References}

1. Moch H, Cubilla AL, Humphrey PA, et al. The 2016 WHO Classification of Tumours of the Urinary System and Male Genital Organs-Part A: Renal, Penile, and Testicular Tumours. Eur Urol 2016;70:93-105.

2. McNeil JC, Corbett ST, Kuruvilla S, et al. Metanephric adenoma in a five-year-old boy presenting with chyluria: case report and review of literature. Urology 2008;72:545-7.

3. Davis CJ Jr, Barton JH, Sesterhenn IA, et al. Metanephric adenoma. Clinicopathological study of fifty patients. Am J Surg Pathol 1995;19:1101-14.

4. Drut R, Drut RM, Ortolani C. Metastatic metanephric adenoma with foci of papillary carcinoma in a child: a combined histologic, immunohistochemical, and FISH study. Int J Surg Pathol 2001;9:241-7.

5. Renshaw AA, Freyer DR, Hammers YA. Metastatic metanephric adenoma in a child. Am J Surg Pathol 2000;24:570-4.

6. Blanco LZ Jr, Schein CO, Patel T, et al. Fine-needle aspiration of metanephric adenoma of the kidney with clinical, radiographic and histopathologic correlation: a review. Diagn Cytopathol 2013;41:742-51.

7. Wright CA, van der Burg M, Geiger D, et al. Diagnosing mycobacterial lymphadenitis in children using fine needle aspiration biopsy: cytomorphology, ZN staining and autofluorescence -- making more of less. Diagn Cytopathol 2008;36:245-51.

8. Lerut E, Roskams T, Joniau S, et al. Metanephric adenoma during pregnancy: clinical presentation, histology, and cytogenetics. Hum Pathol 2006;37:1227-32.

9. Jiang $\mathrm{T}, \mathrm{Li} \mathrm{W}$, Lin $\mathrm{D}$, et al. Imaging features of metanephric adenoma and their pathological correlation. Clin Radiol 2019;74:408.e9-17.

10. Bastide C, Rambeaud JJ, Bach AM, et al. Metanephric adenoma of the kidney: clinical and radiological study of nine cases. BJU Int 2009;103:1544-8.

11. Fielding JR, Visweswaran A, Silverman SG, et al. CT and ultrasound features of metanephric adenoma in adults with pathologic correlation. J Comput Assist Tomogr 1999;23:441-4.

12. Bosniak MA, Birnbaum BA, Krinsky GA, et al. Small renal parenchymal neoplasms: further observations on growth. Radiology 1995;197:589-97.

13. Low G, Sahi K, Dhliwayo H. Low T2 signal intensity on magnetic resonance imaging: a feature of minimal fat angiomyolipomas. Int J Urol 2012;19:90-1.

14. Gakis G, Kramer U, Schilling D, et al. Small renal oncocytomas: differentiation with multiphase CT. Eur J Radiol 2011;80:274-8.

15. Xue LY, Lu Q, Huang BJ, et al. Papillary renal cell carcinoma and clear cell renal cell carcinoma: Differentiation of distinct histological types with contrast - enhanced ultrasonography. Eur J Radiol 2015;84:1849-56.

16. Ascenti G, Gaeta M, Magno C, et al. Contrastenhanced second-harmonic sonography in the detection of pseudocapsule in renal cell carcinoma. AJR Am J Roentgenol 2004;182:1525-30.

17. Xue LY, Lu Q, Huang BJ, et al. Differentiation of subtypes of renal cell carcinoma with contrastenhanced ultrasonography. Clin Hemorheol Microcirc 2016;63:361-71.

18. Mueller-Peltzer K, Negrao de Figueiredo G, Graf T, et al. Papillary renal cell carcinoma in contrast-enhanced ultrasound (CEUS) - A diagnostic performance study. Clin Hemorheol Microcirc 2019;71:159-64.

19. Kazmierski B, Deurdulian C, Tchelepi H, et al. Applications of contrast-enhanced ultrasound in the kidney. Abdom Radiol (NY) 2018;43:880-98.

20. Barr RG, Peterson C, Hindi A. Evaluation of indeterminate renal masses with contrast-enhanced US: a 
diagnostic performance study. Radiology 2014;271:133-42.

21. Xu ZF, Xu HX, Xie XY, et al. Renal cell carcinoma: realtime contrast-enhanced ultrasound findings. Abdom

Cite this article as: Zhu D, Zhu L, Wu J, Wu L, Li J, Luo H, Yang K, Liu G, Quo X, Dong F, Xu J. Metanephric adenoma: association between the imaging features of contrast-enhanced ultrasound and clinicopathological characteristics. Gland Surg 2021;10(8):2490-2499. doi: 10.21037/gs-21-437
Imaging 2010;35:750-6.

(English Language Editor: J. Jones) 